\title{
Investigating the performance of an airblast pressure swirl atomizer
}

\author{
Dilyan Kamenov*1, Lydia Achelis ${ }^{1}$, Volker Uhlenwinkel ${ }^{2}$, Udo Fritsching ${ }^{1,2}$ \\ ${ }^{1}$ Particles and Process Engineering, University of Bremen, Bremen, Germany \\ ${ }^{2}$ Leibniz Institute for Materials Engineering IWT, Bremen, Germany \\ ${ }^{*}$ Corresponding author email: d.kamenov@iwt.uni-bremen.de
}

\begin{abstract}
The current contribution investigates the atomization of a liquid with the help of a novel atomizer design. The pressure gas atomizer (PGA) consists of a combination of a pressure swirl nozzle and a concentric gas nozzle that offers advantages compared to conventional techniques when liquids with complex rheological properties, i.e. metal melts, need to be atomized. As the liquid phase exits the pressure swirl nozzle it is being spread out as a concentric film in the form of a hollow cone with a film thickness of a few hundred micrometres, depending on the liquid outlet diameter. This film already may disintegrate on its own due to instabilities, forming large ligaments or droplets. An annular gas nozzle, mounted underneath the pressure swirl nozzle, induces secondary atomization of the liquid film resp. ligaments by impinging a gas stream on the liquid phase. To be able to draw conclusions on the advantages of the new atomizer design, a conventional close coupled atomizer, which is widely used for atomizing metal melts, has been investigated in comparison. The geometrical parameters varied for both atomizers are the outlet diameter and the number of tangential inlets of the pressure swirl nozzle. The drop size distribution and the sauter mean diameter have been measured within the atomization of a model liquid (tap water) at identical gas and liquid mass flow rates in both atomizers by means of a laser diffraction spectrometer.
\end{abstract}

\section{Keywords}

atomization, pressure-swirl atomizer, airblast atomizer

\section{Introduction}

Pressure swirl atomizers (PSA) are widely used to atomize fuel in combustion chambers, for spray-drying purposes and in gas scrubbers as they produce the smallest mean droplet diameters based on the exit orifice dimensions when used as a single phase pressure atomizer [1]. Inside a pressure swirl nozzle the liquid is being accelerated tangentially and axially, the generated centrifugal forces thus result in the liquid exiting the nozzle in the form of a hollow cone film with a thickness of only a few hundred micrometres, depending on the outlet diameter. This characteristic is advantageous in combination with a gas nozzle within the present pressure gas atomizer design, since it is of great importance to spread the liquid as thin as possible in order to obtain the finest spray [2], even more so when dealing with liquids with high viscosities and surface tensions [3] like metal melts.

Apart from some studies that showed the suitability for the generation of metal powders $[4,5]$ this particular conjunction of nozzles has not been studied extensively so far.

An established design for production of fine metal powders is the close coupled atomizer (CCA) [6]. It consists of a circular plain orifice outlet for the melt feed surrounded by a ring slit gas nozzle which is mounted above on the same axis. The atomization capabilities of plain orifices are negligable below relative injection pressures of about $150 \mathrm{kPa}$ [1]. Since the injection pressure on the melt is significantly lower than this value, the liquid is primarily disintegrated by the high-velocity gas stream. The process is believed to include the formation of a thin liquid film in the vicinity of the outlet through upward recirculation and radial pressure gradients [7], however, at high mass flow rates the film might not form [8]. 
A comparison between a plain jet and a prefilming atomizer was carried out by Rizk and Lefebvre [3] and concluded that the plain jet atomizer performed considerably worse, even more so at lower gas velocities.

The current contribution ties in at this point with an investigation of both the established and the novel atomizer. The goal is to assess and compare the atomizer performances at different liquid and air mass flow rates and reveal potential advantages of the novel design.

\section{Material and Methods}

\section{Atomizer configurations}

To be able to understand how the flow of both phases in the two atomizer designs develops, the basic structure and associated equations from literature are presented below.

Figure 1 shows a drawing of the pressure swirl airblast atomizer (PGA) and the close coupled atomizer (CCA) utilised in the current contribution.
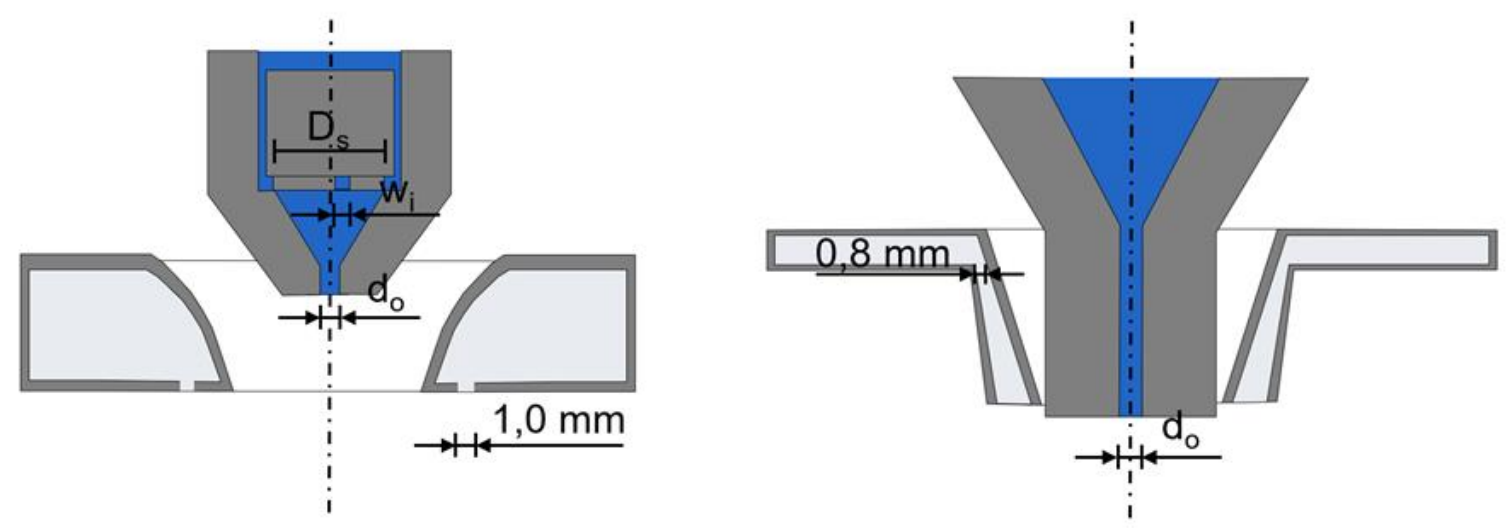

Figure 1. Schematic representation of the PSA (left) and CCA (right) with coaxial gas nozzles

The swirling flow in the pressure swirl atomizer is generated by guiding the liquid through square tangential channels (as opposed to having a built-in spiral solid core) with the width of $w_{i}$ into the swirl chamber with a diameter $D_{s}$. The number of inlets $n_{i}$ was varied between two and four, while orifice diameters $d_{o}$ of $1.0 \mathrm{~mm}$ and $1.5 \mathrm{~mm}$ were investigated. The coaxial annular gas nozzle is fixed below the pressure swirl nozzle to allow for air to be entrained, and consists of 20 circular openings with a diameter of $1.1 \mathrm{~mm}$ arranged in a ring with a diameter of $22 \mathrm{~mm}$. The total area of the gas nozzle is $1.90 \cdot 10^{-5} \mathrm{~mm}^{2}$

The thickness of the generated hollow cone $t$ can be estimated as [9]:

$$
t^{2}=\frac{1560 \dot{m}_{L} \mu_{L}}{\rho_{L} d_{o} \Delta P_{L}} \frac{1+\left(d_{o}-2 t\right)^{2} / d_{o}^{2}}{\left(1-\frac{\left(d_{o}-2 t\right)^{2}}{d_{o}^{2}}\right)^{2}} .
$$

The spray cone half-angle $\theta$ for this specific pressure swirl nozzle can be calculated as [10]

$$
2 \theta=6\left(\frac{n_{i} \cdot\left(w_{i}\right)^{2}}{D_{s} \cdot d_{o}}\right)^{-0,15}\left(\frac{\Delta p_{L} d_{o}^{2} \rho_{L}}{\mu_{L}^{2}}\right)^{0.11}
$$

Equation 2 does not consider the influence of the tangential inlet number on possible velocity changes inside the swirl chamber, $n_{i}$ is used to calculate the total inlet area, which according to the equation has a negative effect on spray cone angle. 
Rashid et. Al. [11] examined the influence of the number of tangential inlets into a pressure swirl nozzle at otherwise identical geometries, i.e. swirl chamber diameters and inlet area. The authors found that an increase in the number of inlets produces wider spray cones with the effect being more significant at higher injection pressures. This observation was explained with an increase of tangential velocity within the swirl chamber.

The plain orifice diameter $d_{o}$ of the close coupled atomizer was varied between 1.0 and 1.5 $\mathrm{mm}$, in accordance to the diameters used within the pressure swirl nozzle. The ring slit gas nozzle has a width of $0.8 \mathrm{~mm}$ at the narrowest point, resulting in a total area of $4.62 \cdot 10^{-5} \mathrm{~mm}^{2}$. Due to the complex interaction between both phases in a close coupled setup, equations that describe phenomenology, e.g. film thickness, are not readily available. However, an important dimensionless number in all two-phase atomization processes is the mass flow ratio of the phases. In the case of the here examined substances this would be the air to liquid ratio $(A L R)$ :

$$
A L R=\frac{\dot{m}_{A}}{\dot{m}_{L}}
$$

In general, the relationship between atomizer performance, i.e. $S M D$, and $A L R$ can be expressed in the form of a power law:

$$
S M D=c \cdot A L R^{x} .
$$

Since higher air to liquid ratios result in smaller sauter mean diameters, the exponent $x$ in Equation $\mathbf{4}$ is expected to be negative. In order for Equation 4 to be dimensionally correct the constant $c$ must have the dimension of a length and can be interpreted as a mean diameter.

The span of the droplet distributions can be expressed with the help of characteristic drop diameters $d_{i}$ such that i\% of the total liquid volume is in droplets of smaller diameter. Typically, $\mathrm{i}$ is chosen as 10,50 and 90, leading to the following expression for the span:

$$
\operatorname{span}=\left(d_{90}-d_{10}\right) / d_{50} .
$$

\section{Experimental setup}

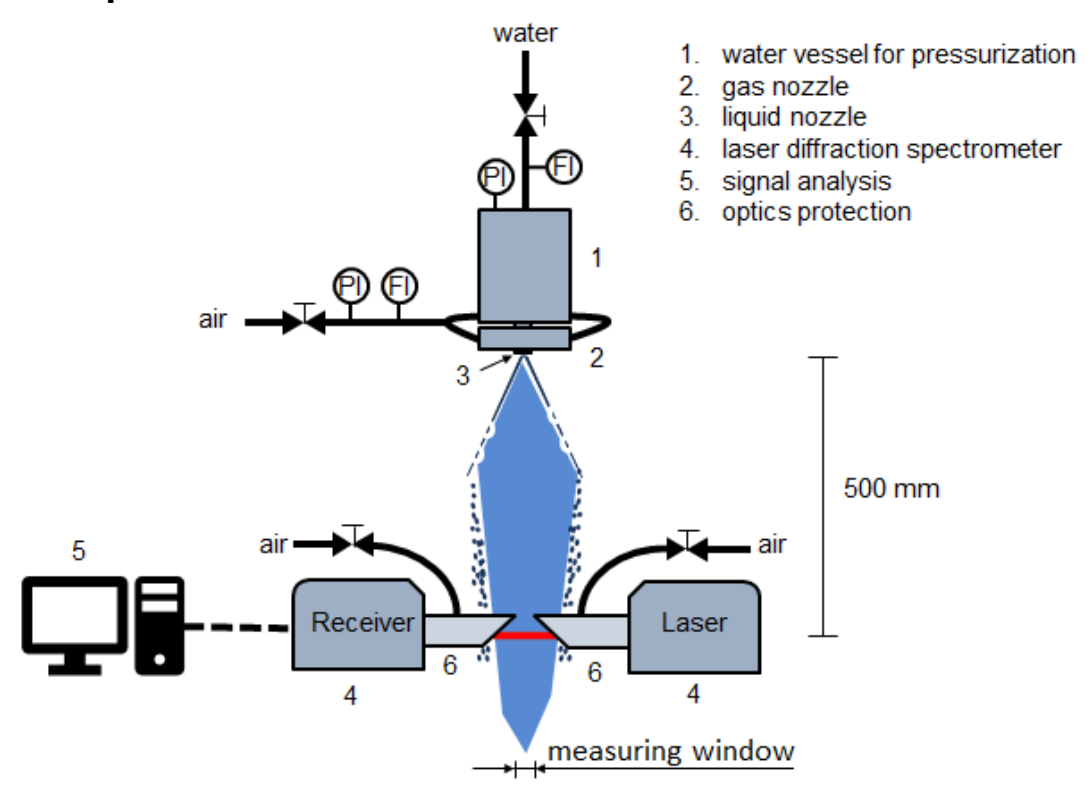

Figure 2. Schematic representation of the experimental setup 
Figure 2 shows the experimental setup, consisting of the atomizer setup with a water vessel for the pressurization (1), the gas nozzle with two air inlets fixed below (2) and the liquid nozzle on the same axis (3). The pressure and mass flow of the liquid and gas is measured. The laser diffraction spectrometer (4) is a HELOS/KR-VARIO by Sympatec, Germany, with a receiver lens with a focal length of $1750 \mu \mathrm{m}$, allowing for a maximum distance between laser and receiver of $566 \mathrm{~mm}$, of which was taken full advantage. The measuring signal is evaluated with the help of the included software based on the Fraunhofer theory. The vertical distance between the edge of the atomizer and laser measurement plain is $500 \mathrm{~mm}$ to ensure that the atomization process is complete and the droplets are spherical. Since two-phase atomizers tend to produce dense sprays, which in turn obscure the incident laser light and facilitate multiple light scattering that leads to overestimation of the smaller droplet fractions, a portion of the spray had to be cut out with the help of cylindrical pipes mounted in front of the laser and receiver (6). Preceding investigations showed that by reducing the measuring window to $30 \mathrm{~mm}$ for the lower gas flow rates and $10 \mathrm{~mm}$ for the highest gas flow rates provided representative measurements while reducing the obscuration below $50 \%$, at which point the effect of multiple scattering should be negligible [12]. Furthermore, the cylindrical pipes prevent droplets from landing on the optics, which would produce measurement errors. Some low velocity air had to be introduced into the pipes as to deflect droplets that divert into the pipes.

Table 1 - Mass flow rates of the gas nozzles and the different PSA geometries

\begin{tabular}{c|cc}
\hline & min. mass flow rate $\left[\mathrm{g} \cdot \mathrm{s}^{-1}\right]$ & max. mass flow rate $\left[\mathrm{g} \cdot \mathrm{s}^{-1}\right]$ \\
\hline gas nozzles & 7.1 & 21.9 \\
PGA d $=1 \mathrm{~mm}, 2$ inlets & 6.2 & 11.1 \\
PGA d $=1 \mathrm{~mm}, 4$ inlets & 7.1 & 12.8 \\
PGA d $=1.5 \mathrm{~mm}, 2$ inlets & 9.8 & 17.2 \\
PGA d $=1.5 \mathrm{~mm}, 4$ inlets & 11.6 & 20.8 \\
CCA d $=1 \mathrm{~mm}$ & 6.2 & 12.8 \\
CCA d & 9.8 & 20.8
\end{tabular}

Table 1 summarizes the mass flow rates of the gas nozzles in both setups as well as the water mass flow rates in the four different geometrical configurations of the PSA and the two respective configurations of the CCA. The liquid flow rate of the two CCA configurations was varied within the minimum and maximum flow rates of the corresponding PSA, e.g. the minimum mass flow rate of the $1 \mathrm{~mm}$ CCA is the minimum mass flow rate of the PSA with 1 $\mathrm{mm}$ orifice diameter and 2 inlets and the maximum is the maximum mass flow rate of the PSA with $1 \mathrm{~mm}$ orifice diameter and 4 inlets.

The minimum water mass flow rate in all geometrical configurations of the PSA corresponds to an injection pressure of $150 \mathrm{kPa}$, the maximum mass flow rate was achieved with an injection pressure of $550 \mathrm{kPa}$. The injection pressures in the CCA nozzles are significantly lower than the pressures needed for the same water mass flow rates in the PSA. As mentioned in the Introduction, liquid injection pressures in close coupled melt atomization are generally low to ensure maximum relative velocities between both phases since it is a coflowing design. Furthermore, the gas pressures employed when atomizing melts with a CCA are significantly higher than the pressures that were reached in the current investigation. This is owed to the fact that the area of the ring slit gas nozzle of the CCA is almost three times bigger than the area of the gas nozzle of the PSA. However air to liquid ratios of up to 3.5 were examined, which is well within the air to liquid ratios needed in close coupled melt atomization [6]. 


\section{Results and Discussion}

In the following the influence of different gas and water mass flow rates, which can be found in Table 1, on sauter mean diameter for both atomizer designs are shown and compared. Furthermore, a closer look at the dependency of both designs on ALR is given thereafter.

\section{Influence of gas and water mass flow rate on sauter mean diameter}
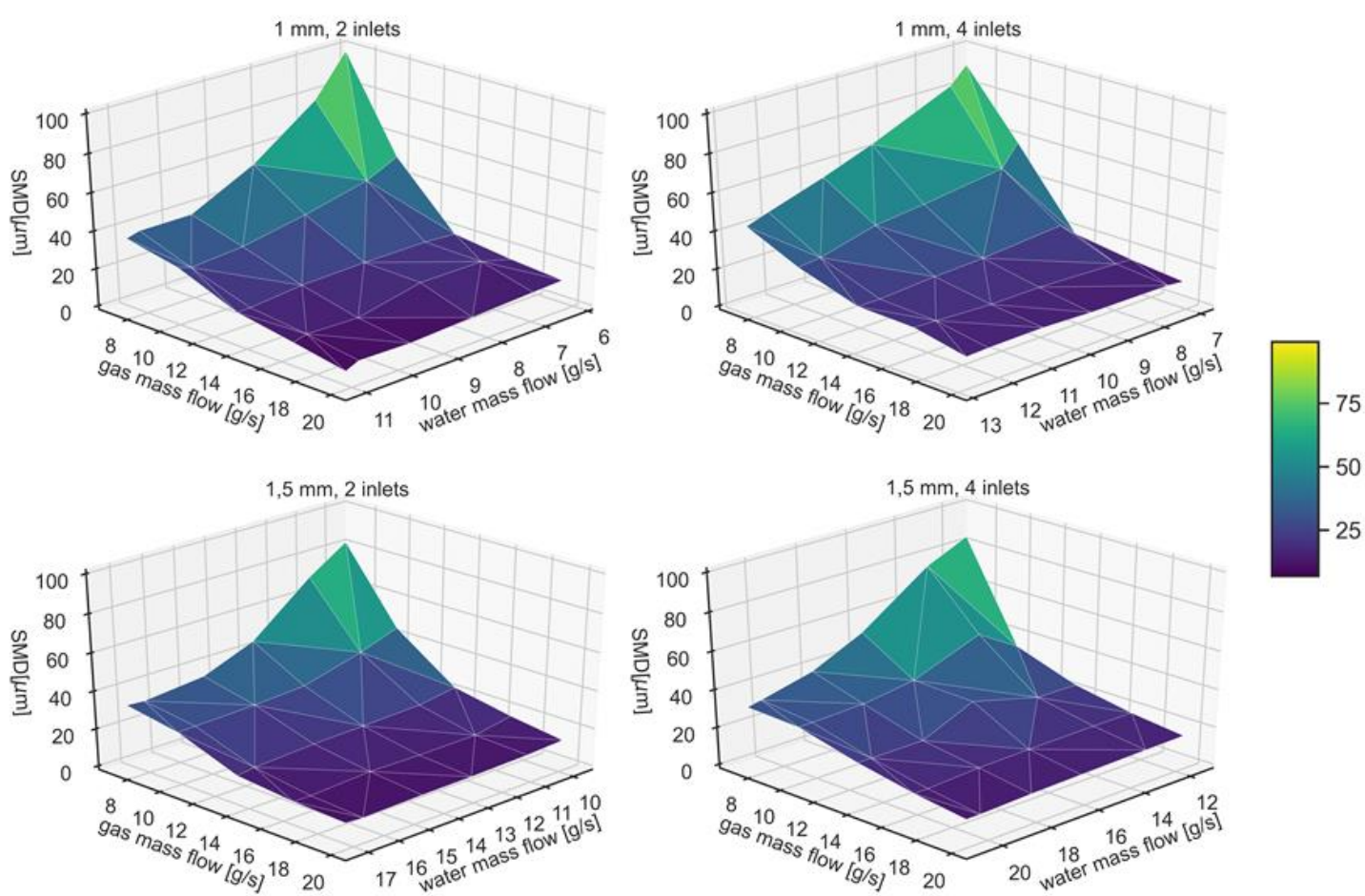

Figure 3. SMD at various air and water mass flows for the different geometrical configurations of the PGA

Figure 3 shows the SMD of the PGA at various air and water mass flow rates for the four geometrical configurations. In general, the SMD decreases with increasing air mass flow rate, as well as with increasing water flow rate. Because of this phenomenon the largest mean diameters can be observed at the lowest gas and water flow rates, which do not amount to the lowest $A L R$, with values for the $S M D$ being between 80 and $100 \mu \mathrm{m}$. Another observation to be made is that at the highest gas flow rates in all four geometries the mean diameter is virtually independent of the liquid flow rate. At those process conditions the lowest SMD of 15 $\mu \mathrm{m}$ were measured.

The decrease of the SMD with increasing gas mass flow at a constant water mass flow is to be expected because of the increasing ALR, whereas the decrease in mean droplet size with increasing water mass flow rate (at a constant gas flow rate) is surprising at first glance, because the air to liquid ratio decreases. An explanation for this effect is that the thickness of the hollow cone decreases according to Equation 1 resulting in smaller SMD. As an example, the prediction for the hollow cone thickness at the lowest water flow rate in the $1.5 \mathrm{~mm} 2$ inlets PGA is $383 \mu \mathrm{m}$, while the highest mass flow rate yields a thickness of $315 \mu \mathrm{m}$. The decrease in film thickness is however not significant enough to explain the high drop in mean diameter at the lowest gas mass flow rates, which can be observed in all geometries.

Based on Equation 2 the hollow cone angle increases with increasing injection pressure thus improving the momentum exchange between both phases. Furthermore, an increase in orifice diameter and inlet ports should lead to larger cone angles at the same pressure differentials, according to Equation 2 and as found by Rashid et. Al. [11].

This effect could also compensate for the increased thickness within the larger orifices and number of inlet ports and explain the overall similar SMDs observed in all configurations. 
The mean span (s. Equation 5) of the droplet distributions can be given as 1.92 for the $1 \mathrm{~mm}$, 2 inlets, 1.98 for the $1 \mathrm{~mm}, 4$ inlets, 1.91 for the 1.5, 2 inlets and 1.93 for the 1.5, 4 inlets configurations.
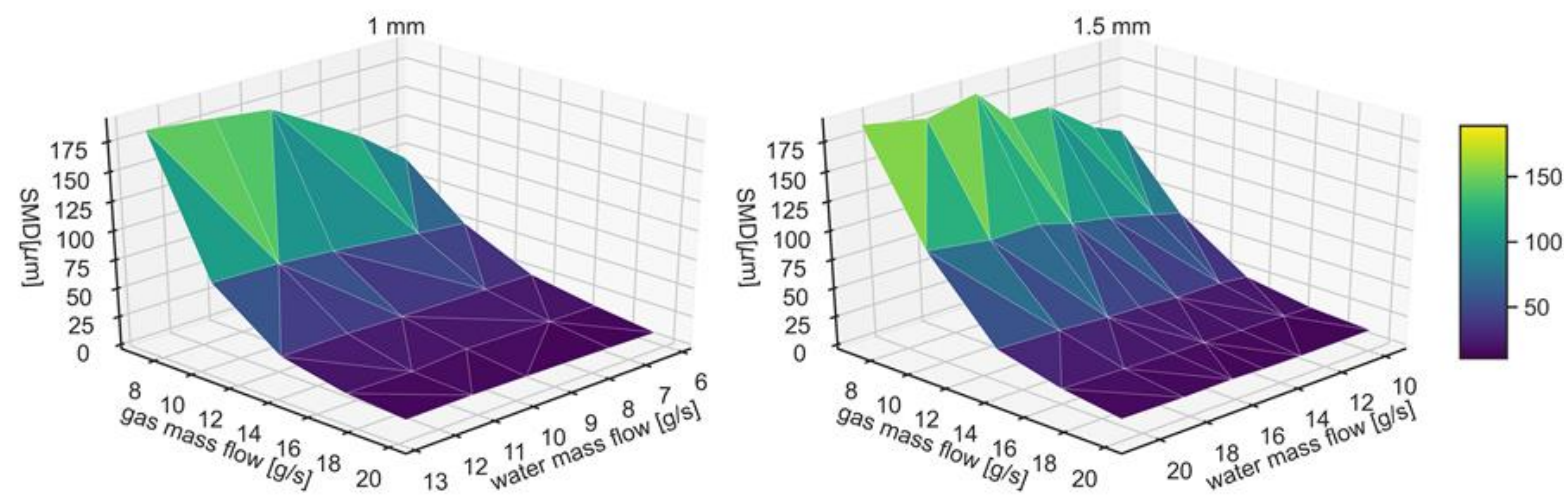

Figure 4. SMD at various air and water mass flows for the two geometrical configurations of the CCA

Figure 4 shows the SMD of the two examined CCA configurations at various gas and water mass flow rates. As can be seen the SMD decreases with increasing gas mass flow and increases at higher water mass flow rates. A comparison of both orifice diameters shows that the mean diameters are lower for the $1 \mathrm{~mm}$ orifice owing to the higher $A L R$ with the difference being negligible at the two highest gas flow rates. A significant underperformance at low gas mass flow rates can be observed compared to the PGA, the mean diameters produced by the CCA at the lowest $A L R$ are almost six times higher than the mean diameters measured within the PGA configuration at the same $A L R$. The lacking performance at low mass flow ratios is in agreement with the findings in [3]. As the gas mass flow rate increases the difference becomes less notable and at the highest flow rates both atomizer designs exhibit similar mean diameters of the order of $15 \mu \mathrm{m}$.

The mean span of the droplet size distributions within the $1 \mathrm{~mm} \mathrm{CCA} \mathrm{configuration} \mathrm{is} \mathrm{slightly}$ lower than the ones of the PGA configurations with the value being 1.9 . The $1.5 \mathrm{~mm}$ orifice diameter CCA however produces significantly wider distributions with a span of 2.1 on average.

\section{Dependency of sauter mean diameter on air to liquid ratio}

To evaluate the influence of the air to liquid ratio on sauter mean diameter (SMD) the coefficients in Equation 4 were determined along the lines of constant water mass flow rate in Figure $\mathbf{3}$ and Figure $\mathbf{4}$ through means of regression analysis. Figure $\mathbf{5}$ shows the exponents of Equation 4 for the PGA geometries. The mean for the coefficients of determination lies above 0.95 , indicating that Equation 4 offers a good basis for a fit.

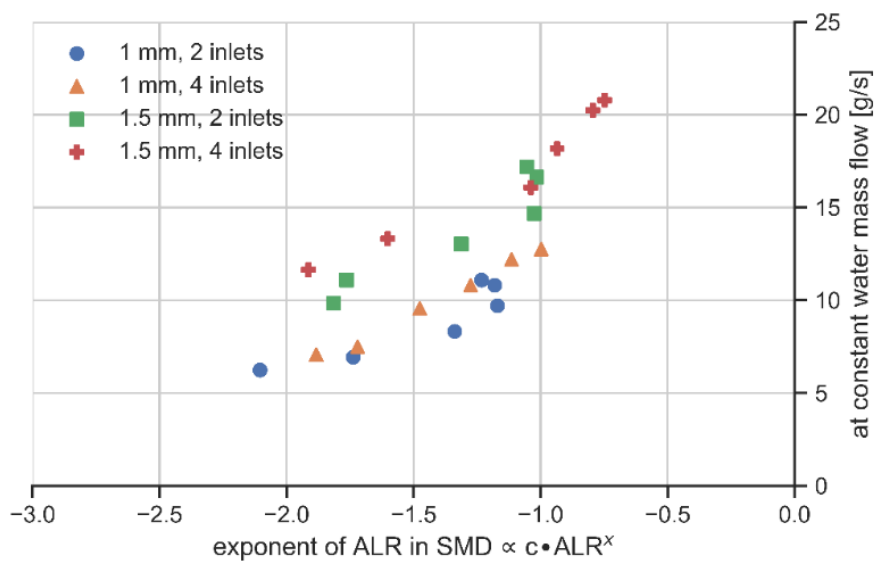

Figure 5. Influence of ALR on SMD at constant water mass flow rates for the different PGA geometries 
The exponents in all geometrical configurations of the PGA are negative, the SMD decreases with increasing $A L R$, which at constant water mass flow rate can only be achieved by increasing the gas flow rate.

Furthermore, the absolute value of the exponent decreases at higher constant water flow rates throughout all four geometries, falling as low as -0.74 within the $1.5 \mathrm{~mm} 4$ inlets PGA. This is a significant result for this kind of atomizer since the gas flow rate was varied in the same range, thus the $A L R$ is significantly higher for the $1 \mathrm{~mm} 2$ inlets nozzle than the $1.5 \mathrm{~mm} 4$ inlets nozzle. The decrease in the absolute value of the exponent indicates that the nozzle becomes less reliant on high air to liquid ratios. An explanation for this effect could be the increasing hollow cone angle, which leads to better momentum exchange of the phases, as discussed in the previous section.

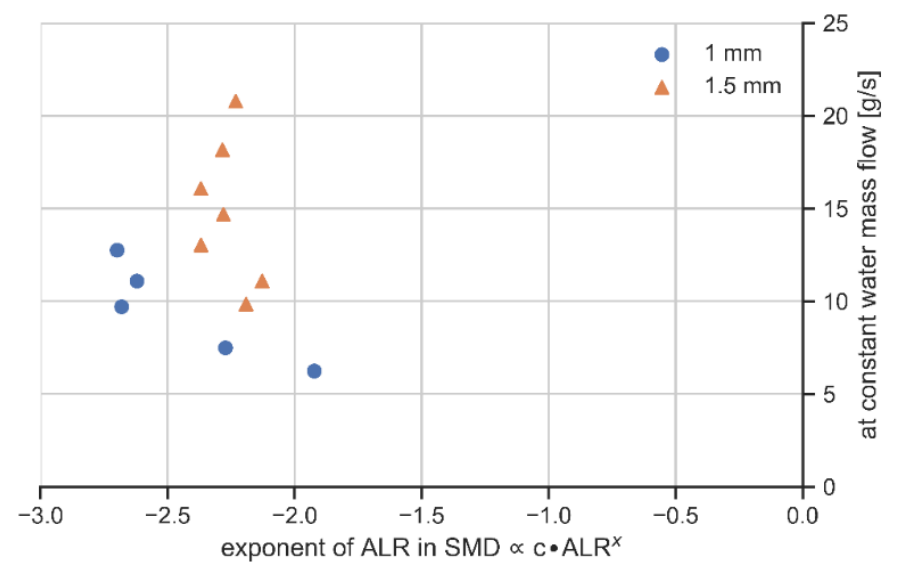

Figure 6. Influence of ALR on SMD at constant water mass flow rates for the two CCA geometries

Figure 6 shows the coefficients for the two CCA geometries, the approach from Equation 4 is also found to be fitting since the coefficients of determination are above 0.98 . As can be seen the exponent is negative for all regressions, thus the $S M D$ decreases with an increase in $A L R$, which was also observed for the PGA. However, the absolute value of the exponent increases at higher constant water mass flow rates in contrast to Figure 5. This implies that the CCA becomes more reliant on ALR while increasing the liquid output. Therefore, the PGA is a more effective airblast design, which utilizes the air better, since the absolute value of the exponent for the highest water flow rate in the $1.5 \mathrm{~mm} 4$ inlets PGA is noticeably lower than the exponent in the $1.5 \mathrm{~mm} \mathrm{CCA}$ at this value.

\section{Conclusions}

The PGA novel atomizer design consisting of a combination of a pressure swirl nozzle and a concentric gas nozzle performs significantly better, i.e. lower $S M D$, than the established close coupled atomizer (CCA) at lower air to liquid ratios. Furthermore, the dependency of the PGA on high mass flow ratios decreases with decreasing $A L R$ in contrast to the findings of the CCA. The better atomizer performance can be explained with the improved momentum exchange between the liquid and gas phase owed to the increase in hollow cone angle at increasing injection pressures, orifice diameters and inlets into the swirl chamber of the pressure swirl atomizer.

In terms of droplet size distributions, the PGA configurations offer similarly wide distributions compared to the $1 \mathrm{~mm}$ CCA geometry and noticeably narrower distributions than the $1.5 \mathrm{~mm}$ CCA nozzle on average. 


\section{Acknowledgments}

The project is funded by the German Federal Ministry for Economic Affairs and Energy (BMWi) within the "Zentrales Innovationsprogramm Mittelstand" (funding number: 16KN078702). The authors are grateful for the financial support and to the project partners Indutherm Gießtechnologie GmbH (Walzbachtal, Germany) and DHCAE Tools (Krefeld, Germany) for the collaboration.

\section{Nomenclature}

$A L R \quad$ air to liquid ratio [-]

CCA close coupled atomizer

$D_{s} \quad$ swirl chamber diameter [m]

$d_{0} \quad$ orifice diameter [m]

$d_{i} \quad$ diameter such that $i \%$ of the total liquid volume is in droplets of smaller diameter [m]

$\dot{m}_{A} \quad$ air mass flow rate $\left[\mathrm{kg} \cdot \mathrm{s}^{-1}\right]$

$\dot{m}_{L} \quad$ liquid mass flow rate $\left[\mathrm{kg} \cdot \mathrm{s}^{-1}\right]$

$n_{i} \quad$ number of inlets of the pressure swirl nozzle [-]

$P S A$ pressure swirl atomizer

$S M D$ sauter mean diameter [m]

span span of the droplet size distribution [-]

$w_{i} \quad$ width of the square tangential inlet channel [m]

$\Delta p_{L} \quad$ pressure difference [Pa]

$\theta \quad$ hollow cone half angle $\left[^{\circ}\right]$

$\mu_{L} \quad$ liquid viscosity $\left[\mathrm{kg} \cdot \mathrm{m}^{-1} \cdot \mathrm{s}^{-1}\right]$

$\rho_{L} \quad$ liquid density $\left[\mathrm{kg} \cdot \mathrm{m}^{-3}\right]$

\section{References}

1. Lefebvre, A.H. and V.G. McDonell, Atomization and sprays. Second Edition ed. 2017, Boca Raton: CRC Press.

2. Lefebvre, A.H. and D. Miller, The development of an air blast atomizer for gas turbine application. 1966: College of Aeronautics.

3. Rizkalla, A.A. and A.H. Lefebvre, The Influence of Air and Liquid Properties on Airblast Atomization. Journal of Fluids Engineering, 1975. 97(3): p. 316-320.

4. $\quad$ Lagutkin, S., et al., Atomization process for metal powder. Materials Science and Engineering A, 2004. 383: p. 1-6.

5. Achelis, L., et al., Spray angle and particle size in the pressure gas atomization of Tin and Tin-Copper alloys. Proceedings of the World Powder Metallurgy Congress and Exhibition, World PM 2010, 2010. 1.

6. Mates, S. and G. Settles, A study of liquid metal atomization using close-coupled nozzles, Part 1: Gas dynamic behavior. Atomization and Sprays - ATOMIZATION SPRAYS, 2005. 15: p. 19-40.

7. Klar, E.F.J.W., Atomization, in Metals Handbook. 1984, American Society for Metals: Metals Park, $\mathrm{OH}$.

8. Ünal, A., Liquid break-up in gas atomization of fine aluminum powders. Metallurgical Transactions B, 1989. 20(1): p. 61-69.

9. Rizk, N.K. and A.H. Lefebvre, Internal flow characteristics of simplex swirl atomizers. Journal of Propulsion and Power, 1985. 1(3): p. 193-199.

10. N.K. Rizk and A.H. Lefebvre, Prediction of Velocity Coefficient and Spray Cone Angle for Simplex Swirl Atomizers. International Journal of Turbo and Jet Engines, 1987. 4(1-2): p. 65-74.

11. Rashid, M.S.F.M., et al., Effect of Inlet Slot Number on the Spray Cone Angle and Discharge Coefficient of Swirl Atomizer. Procedia Engineering, 2012. 41: p. 1781-1786.

12. Triballier, K., C. Dumouchel, and J. Cousin, A technical study on the Spraytec performances: influence of multiple light scattering and multi-modal drop-size distribution measurements. Experiments in Fluids, 2003. 35(4): p. 347-356. 